\title{
Loin Pain Haematuria Syndrome Complicating Nehroptosis: Case Report
}

\author{
Ahmed N Ghanem* \\ Consultant Urological Surgeon, Egypt
}

*Corresponding author: Ahmed N Ghanem, Consultant Urological Surgeon, No1 President Mubarak Street, Mansoura 35511, Egypt

\section{ARTICLE INFO \\ Received: 幽 March 15, 2019 \\ Published: March 22, 2019 \\ Citation: Ahmed N Ghanem. Loin Pain Haematuria Syndrome Complicating Nehroptosis: Case Report. Biomed J Sci \& Tech Res 16(2)-2019. BJSTR. MS.ID.002834.}

Keywords: Nephroptosis; Loin pain haematuria syndrome; IVU; Ancillary imaging

Abbreviations: IVU: Intravenous Urography; CAT: Computer Axial Tomography; MRI: Magnetic Resonance Imaging; DSA: Digital Subtraction Angiography; IR: Isotope Renography; RSD\&N: Renal Sympathetic Denervation and Nephropexy; RGP: Retrograde Pyelography; DSA: Digital Subtraction Arteriography; LPHS: Loin Pain Haematuria Syndrome; SN: Symptomatic Nephroptosis; TIA: Transient Ischaemic Attacks
ABSTRACT

Objective: To report a case in which loin pain haematuria syndrome complicated right Nehroptosis.

Methods: Data belonging to a case of a 25 years old Saudi female in which loin pain haematuria syndrome complicated right nehroptosis is reported. All urological investigation including intravenous urography (IVU), computer axial tomography (CAT), magnetic resonance Imaging (MRI), Digital subtraction angiography (DSA) and isotope renography (IR) were done. A repeat of IVU with erect 15 minutes film diagnosed nephroptosis.

Results: The case initially diagnosed with loin pain and later developed microscopic haematuria. Gross hamaturis occurred 3 years later. All radiological imaging of IVU, CAT, MRI and IR were normal. A repeat of IVU with erect 15 minutes film diagnosed nephroptosis while all other imaging was normal on supine position. The patient was hospitalised on average once every 2 weeks and investigated at multiple centres. Psychological disorders, opiate dependency and/or imaginary pain were thought on repeated admissions to be the cause of her undiagnosed and treated suffering. She underwent unsuccessful nephropexy elsewhere with recurrence of SN and LPHS. She has been on conservative treatment as she refused the new curative surgery for her condition, namely renal sympathetic denervation and nephropexy(RSD\&N) surgery which has proved successful in other cases.

Conclusion: The only way to diagnose SN is by doing IVU with erect film. All other ancillary imaging's were normal being feasible only on supine posture. The case demonstrates the progression of SN to the complication of LPHS which occurred after 3.5 years. The surgery of RSD\&N has proved successful in many other cases, but this patient refused it.

\section{Case Report}

A 25 years old Saudi single female school teacher presented 9 years ago to the haematologist with iron deficiency anaemia (Haemoglobin was $8.3 \mathrm{gr} / \mathrm{l}$. During her stay in hospital, she suffered from bilateral epistaxis. Mucoperiostal elevation cured her epistaxis after 7 bilateral cauterizations had failed to control her nasal bleeding. No bleeding or coagulation abnormalities were detected. She was also reported to have dysmenorrhea, dysurea and right loin pain. Attending physicians excluded genitourinary and other system abnormalities, Loin pain and microscopic haematuria reoccurred. Urological investigations including Urine analysis and culture, full blood count, renal function tests, grayscale ultrasound and intravenous urography (IVU) were repeatedly normal. No cause for her painful haematuria was found for 5 years but it was confirmed to originate from her right kidney on cystoscopy when bleeding was seen spyrting from the right ureteric orifice. Retrograde pyelography (RGP) was initially normal. She had repeated investigations done at various hospitals for her loin pain and haematuria. 
These included computer axial tomography (CAT) scan, magnetic resonance imaging (MRI), digital subtraction arteriography (DSA) and isotope renography which were all normal in supine posture. Psychological disorders, opiate dependency and/or imaginary pain were thought on repeated admissions to be the cause of her undiagnosed and treated suffering. Psychiatric assessment excluded personality disorders and she was denied opiate therapy for years. Five years ago, right nephroptosis was suspected after palpating a mass at the right iliac foss on erect examination which disappeared on lying supine- unless the kidney mass was held down by gripping the loin above it. Nephroptosis was demonstrated on IVU with erect film. The kidney dropped $>3.5$ vertebrae from its normal position to the pelvis. A year later here haematuria progressed to frequent gross episodes of frank bleeding with intractable renal pain requiring frequent hospitalizations. During these attacks she was treated conservatively by fluid therapy, blood transfusions, antibiotics, opiates and bed rest. Thorough gynecological, nephrological, neurospinal, gastrointestinal and haematological investigations were repeatedly normal at various centres.
Two years ago, she underwent right nephropexy elsewhere but ptosis and haemauuria reoccurred. Even now the right kidney remains posted on erect IVU (Figure 1, upper segment). Repeated RGP showed atony and dilatation of right renal pelvis (Figure 1, middle segment- right). Bladder biopsy showed interstitial cystitis. Repeated CAT showed upper pole cyst and normal otherwise (Figure 1, lower segment). MRI was normal. DSA was normal apart from migration of the renal artery towards upper pole (Figure 1, middle segment- left)- being feasible only on supine posture. Right renal biopsy showed mesangial proliferative glomerulonephritis. Left renal biopsy was refused. DTPA isotope renographic study was also normal on supine posture. On repeating this study on sitting up posture, the right kidney showed impaired perfusion and filtration: GFR of 57.5 in the left kidney and 40.5 in the right kidney were demonstrated on supine posture. At sitting up posture, GFR of 63.8 in the left kidney and 22.2 in the right posted kidney were demonstrated. The normal expected value is $104 \mathrm{ml} /$ minute. Such big deficit in differential renal function of the right kidney at posted position occurred in the abscence of organic ureteric stenosis or obstruction.
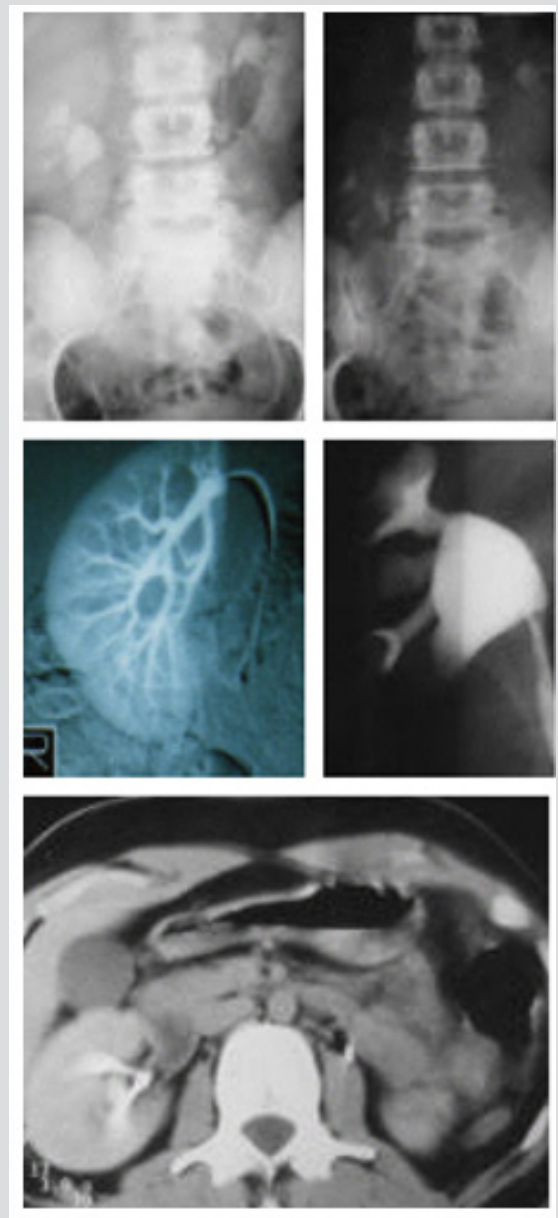

Figure 1: It shows 3 segments of upper, middle and lower.

Note: Upper segment shows IVU with supine film on the left and erect film on the right side. Right nephroptosis of 3 vertebrae is shown despite previous nephropexy. Middle segment shows DSA on the left that normal apart from migration of the renal artery towards upper pole. The RGP is shown on the right side and demonstrates pelvis dilatation. The lower segment shows CAT scan which is normal apart from upper pole cyst. 
These findings suggest diminished right arterial blood flow and GFR on upright posture, possibly due stretch narrowing (uniform stenosia) of the renal artery. Diminished vasculature of the right kidney, as compared to the left, may be observed on DSA- despite being done at supine posture (Figure 3). Frequent crisis of the loin pain haematuria syndrome (LPHS) have continued during the last 4 years, requiring countless hospital admissions. She has been so crippled by her LPHS that she lost her job as school teacher. She carries a bag full of useless medications and a list of prohibited nephrotoxic drugs. She refused surgical treatment of renal sympathetic denervation and nephropexy surgery which has proved successful in other cases.

\section{Discussion}

The natural history of this case was followed up from the initial onset of loin pain to occurrence of recurrent microscopic haematuria, to diagnosis of symptomatic nephroptosis (SN), to the development of LPHS [1]. To my knowledge this is the first case report of LPHS to complicate SN. It is certainly not the last [2]. It demonstrates many of the problems encountered in the diagnosis and therapy of both SN and LPHS. All textbooks do not index SN as it was disparaged long ago [3] but mention LPHS. Chance diagnosis of SN on supine imaging is unlikely. So, SN has become a universally forgotten diagnosis [3]. Vascular anomalies, ischaemic renal scarring and messengial proliferative glomerulonephritid are features of LPHS [1]. Most of these renovascular complications of LPHS are also documented in SN [4-7]. Despite known overinvestigations, no erect imaging was ever done in LPHS. Previous reports on erect artieriography [5-7] and isotope renography [7] demonstrated these complications in SN. Recurrent stretch of renal vessels in erect posture causes renal artery elongation and narrowing in the presence or absence of stenostic lesions [5].

Transient ischaemic attacks (TIA) of renal pain or "renal angina" occur in the absence of organic lesions for years. Chronicity establishes fibro-muscular dysplasia [5,6] manifesting with the organic ischaemic renal complications of LPHS [1,2] and pelviureteric atony- as shown in the case reported here. To salvage a kidney with such irreversible damage by sympathectomy and/ or auto renal transplantation is extremely hard, and $75 \%$ of cases end up with nephrectomy [8]. Our patient and I are resenting nephrectomy which though cures pain and haematuria and saves what is left of her miserable life is hazadous. This is because of a possible involvement of the left kidney too. Conservative treatment is neither effective nor safe. My patient, being a Muslim, has found relief and comfort in her prayers, particularly at so good position or the knee-chest position. The spiritual and gravitational benefits of this position are obvious. This is an example of how the weak and helpless find power to resist an unbearable situation. Other patients like her become depressed by the prolonged undiagnosed and untreated suffering [1,4] and may even commit suicide [3].
Her painful episodes of haeematuria are also known "Dietl crisis" of SN, who also advised knee-chest position for temporary relief. This tragic case represents the tip of an iceberg. Experience at King Khaled Hospital in Najran Saudi Arabia with 190 cases suffering from SN, of whom LPHS complicated SN in 36 (18.9\%) of patients $[8,9]$. This figure represents cases with gross haematuria only. It is higher when cases with microscopic haematuria are included. Making an early diagnosis of SN by erect IVU may upright issues and brings relief to the unfortunate sufferers. Based on recent reports, SN has proved to be a preventable cause of LPHS when the correct surgical procedure is done timely. The surgery of renal sympathetic denervation and nephropexy has proved curable for Both SN and LPHS. Both nephropexy for SN and renal sympathetic denervation of the LPHS were reported separately $[10,11]$. Modern favourable results of renal sympathetic denervation and nephropexy (RSD\&N) have proved 100\% success rate for both SN and LPHS $[8,9]$. Nephroptosis was disparaged and nephropexy was abandoned $>70$ years ago, both have been deleted from surgical and urological textbooks and have become universally forgotten. This is because of the many problems in diagnosis and therapy that have been recently addressed and resolved $[10,11]$.

\section{References}

1. Little PJ, Sloper JS, de Wardner HE (1967) A syndrome of loin pain haematuria associated with disease of the peripheral renal arteries. Q J Med 36(142): 253-259.

2. Ghanem AN (2002) Features and complications of symptomatic nephroptosis causing the loin pain haematuria syndrome: Preleminary report. Saudi Med J 23(2): 197-205.

3. Hoenig DM, Hemal AK, Shalhav AL, Clayman RV (1999) Nephroptosis: A "disparaged" condition revisited. Urology 54(4): 590-596.

4. Burford CE (1946) Nephroptosis with coexisting renal lesions. J Urol 55: 220-224.

5. Kaufman JJ, Hanafee W, Maxwell MH (1964) Upright renal arteriography in the study of renal hypertension. JAMA 187: 977-980.

6. Stoll HG (1970) Indications of Nephropexy with special reference to the renovascular aspects of ptosis. Der Urologe A 9(3): 114-117.

7. O Reilly PH, Pollard AJ (1988) Nephroptosis: a cause of renal pain and a potential cause of inaccurate split renal function determination. Br J Urol 61(4): 284-288.

8. Ghanem SA and Ghanem AN (2016) Prospective Observational Study on Loin Pain Hematuria Syndrome Complicating Symptomatic Nephroptosis and the Results of Renal Sympathetic denervation and Nephropexy Surgery. J J Nephro Urol 3(1): 024.

9. Ghanem Salma A, Ghanem Khalid A, Pindoria Nisha, Ghanem Ahmed N (2017) Loin Pain and Haematuria Syndrome (LPHS) Linked to Symptomatic Nephroptosis (SN) and Revealing Pedicle Stretch Causing Neuro-Ischaemia Using the New IVU 7 Sign. Exp Tech Urol Nephrol 1(1): 2-6.

10. Hahn E (1881) Die operative Behandlung der beweglichen Niere Durch fixation. Zintralbl Chir 29: 449-452.

11. Blacklock ARE (1989) Renal denervation with releasing renal capsule incision in the loin pain haematuria syndrome. Br J Urol 64(2): 203-204. 


\section{ISSN: 2574-1241}

DOI: 10.26717/BJSTR.2019.16.002834

Ahmed N Ghanem. Biomed J Sci \& Tech Res

(C) (P) This work is licensed under Creative

Submission Link: https://biomedres.us/submit-manuscript.php

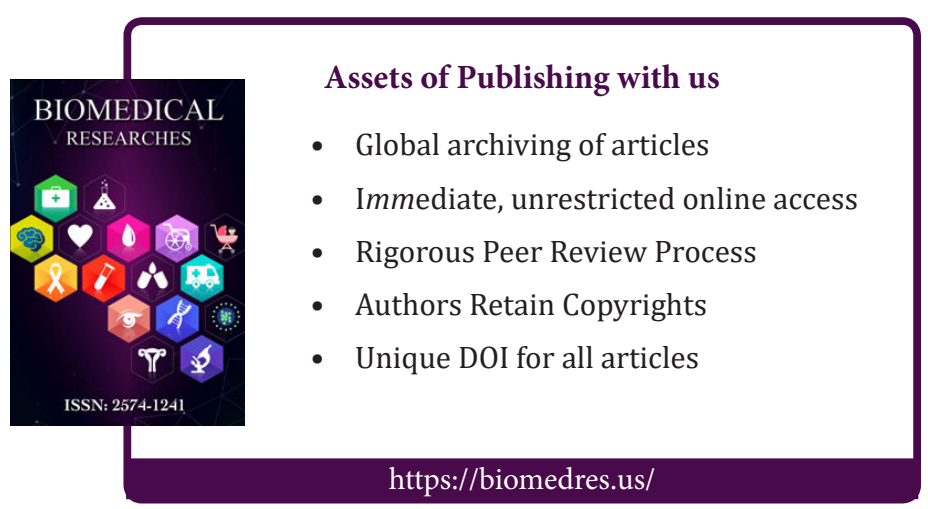

